


\section{Nordic Education Key Data 2012}

ANP 2012:733 


\section{Nordic Education - Key Data 2012}

ISBN 978-92-893-2367-3

DOI http://dx.doi.org/10.6027/ANP2012-733

ANP 2012:733

(C) Nordic Council of Ministers

Cover design: Jette Koefoed

Photos: ImageSelect

Print: Scanprint, Viby

Copies: 2000

Printed in Denmark

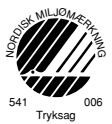

Main suppliers of data: Nordic database, OECD, Eurostat. Editor: Klaus Munch Haagensen, Statistics Denmark.

\section{Nordic Council of Ministers}

Ved Stranden 18

DK-1061 Copenhagen K

Telefon: +4533960200
The Nordic Council

Ved Stranden 18

DK-1061 Copenhagen K

Telefon: +45 33960400

www.norden.org

\section{Nordic co-operation}

Nordic co-operation is one of the world's most extensive forms of regional collaboration, involving Denmark, Finland, Iceland, Norway, Sweden, and the Faroe Islands, Greenland, and Åland.

Nordic co-operation has firm traditions in politics, the economy, and culture. It plays an important role in European and international collaboration, and aims at creating a strong Nordic community in a strong Europe.

Nordic co-operation seeks to safeguard Nordic and regional interests and principles in the global community. Common Nordic values help the region solidify its position as one of the world's most innovative and competitive. 


\section{Preface}

This is the second edition of a booklet, which was published for the first time in 2010, entitled "The Nordic Countries in Educational Key Figures 2010". Like its predecessor, this edition “Nordic Education - Key Data 2012", contains up-to-date tables and diagrams on Nordic education. The booklet has been prepared for everyone interested in Nordic Education statistics, both inside and outside the Nordic region.

The Nordic figures are in most tables and diagrams compared with a number of other countries to achieve a global perspective of education in the Nordic countries. Since the Nordic region is a part of Europe, the EU is a natural and important entity with which to compare figures. The Netherlands has been selected owing to its many similarities with the Nordic countries as regards both size and structure of the welfare state. Asia is represented by Korea and Japan because they both rank among the top ten in all Pisa studies. The OECD represents many of the industrialized countries, while the United States has been chosen since it is often used for benchmarking in similar studies.

The booklet has been prepared by Statistics Denmark and is published by the Nordic Council of Ministers.

The booklet, together with the underlying statistical material, is also available at the website www.norden.org/publications 


\section{Contents}

Preface 3

Glossary 5

Summary 6

Day care $\quad 7$

Basic school 9

Upper secondary education $\quad 14$

Higher education 19

Students abroad 22

Attained education $\quad 25$

Adult education 28

Expenditure in the education system 29

The Nordic education systems

Throughout the book the following symbols and country codes are used:

Symbols

Nil

Less than half

Not applicable

Data not available

\section{Country codes}

Denmark: DK Iceland:IS

Faroe Islands: FO Norway: NO

Greenland: GL

Finland: $\mathrm{FI}$

Sweden: SE

Åland: AX

Korea: KO

Netherlands: NL

Japan: JP
USA: USA

EU-21: Austria, Belgium, the Czech Republic, Denmark, Estonia, Finland, France, Germany, Greece, Hungary, Ireland, Italy, Luxembourg, the Netherlands, Poland, Portugal, Slovenia, the Slovak Republic, Spain, Sweden and the United Kingdom.

EU-27: The 27 member states of the European Union.

To describe the different countries in the figures the following colours are used:

Denmark:

Faroe Islands:

Greenland:

Finland:

Åland:
Iceland:

Norway:

Sweden:

EU:

Korea:
Netherlands:

Japan:

USA:

OECD: 
Day care / pre-school

Day care / pre-school

Förskola

Päiväkoti

Primary level

Basic school

- Pre-school class

- Primary school

- Lower secondary Grundskola

Perusopetus

- Förskoleklass

Esiopetus

- Lågstadiet

- Högstadiet

Upper secondary level

Upper secondary

- General

- Vocational

Gymnasium

- Generell

- Yrkesförberedande program
Lukio

- Yleinen

- Ammatillinen

Tertiary level

Higher education Universitet och högskola Korkea-aste

Adult education

Adult education 


\section{Summary}

Children, pupils and students by level of education. 2010

\begin{tabular}{|c|c|c|c|c|c|}
\hline & DK & FI & IS & NO & SE \\
\hline \multicolumn{6}{|c|}{1000 persons } \\
\hline \multicolumn{6}{|l|}{ Day care } \\
\hline Total & 321 & 180 & 20 & 277 & 476 \\
\hline $0-2$ years & 129 & 50 & 8 & 101 & 158 \\
\hline $3-5$ years & 192 & 131 & 13 & 175 & 318 \\
\hline \multicolumn{6}{|c|}{ Primary education } \\
\hline Total & 116 & 71 & 4 & 65 & 256 \\
\hline Men & 61 & 37 & 2 & 34 & 119 \\
\hline Women & 55 & 34 & 2 & 32 & 137 \\
\hline \multicolumn{6}{|c|}{ Upper secondary education } \\
\hline Total & 247 & 392 & 26 & 266 & 564 \\
\hline Men & 126 & 189 & 13 & 141 & 267 \\
\hline Women & 121 & 203 & 13 & 126 & 296 \\
\hline \multicolumn{6}{|c|}{ Tertiary education } \\
\hline Total & 204 & 308 & 19 & 226 & 469 \\
\hline Men & 86 & 142 & 7 & 89 & 192 \\
\hline Women & 119 & 166 & 12 & 137 & 277 \\
\hline
\end{tabular}

Source: Nordic database. Day care: Iceland 2009 data. Education: Sweden 2008 data.

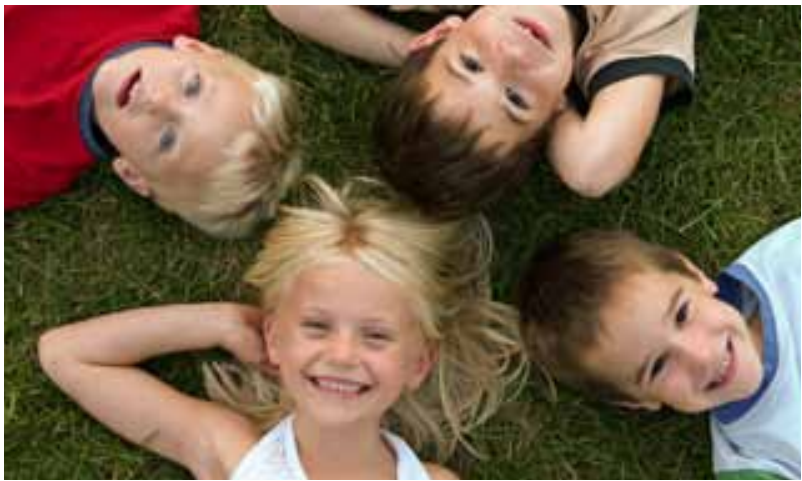


Children in day care in the age group 0-2 years

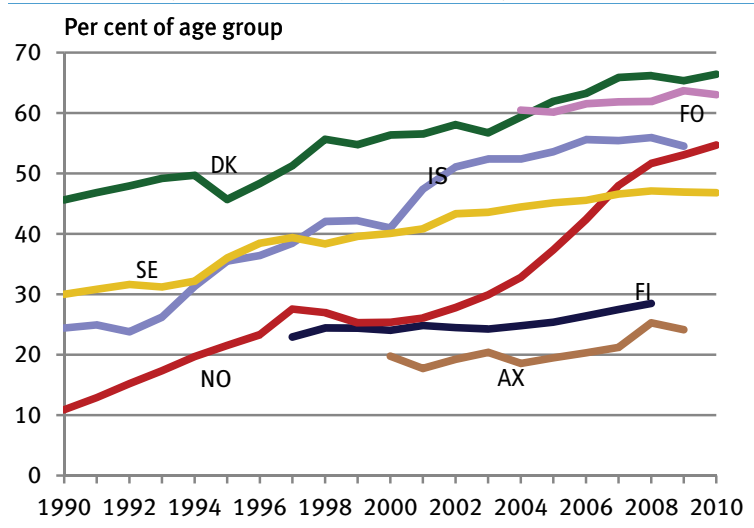

Source: Nordic database.

Children in day care in the age group 3-5 years

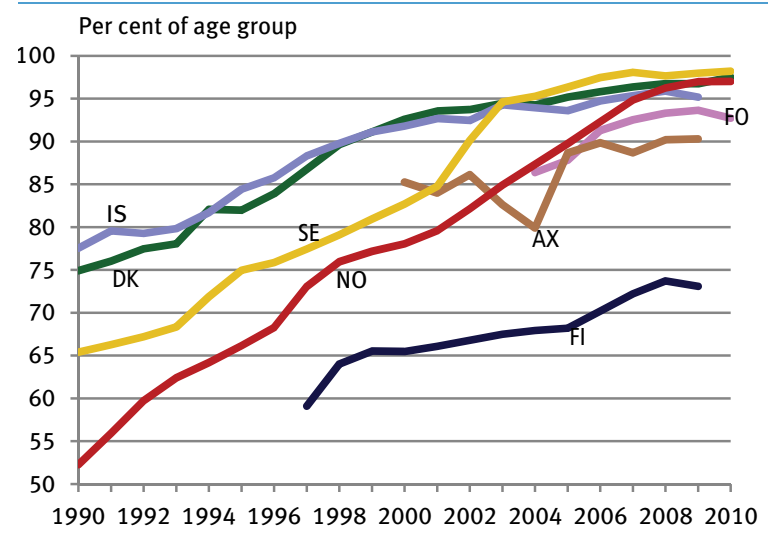

Source: Nordic database.

Nordic Education - Key Data 2012 
Children in day care. 2010

\begin{tabular}{lrr}
\hline & $\begin{array}{r}\text { Age group } \\
\text { 0-2 years }\end{array}$ & $\begin{array}{r}\text { Age group } \\
3-5\end{array}$ \\
\hline yer cent of age group & & \\
\hline Denmark & & \\
Faroe Islands & 66.4 & 97.5 \\
Finland & 63.0 & 92.7 \\
Åland & 27.2 & 73.3 \\
Iceland & 24.1 & 90.3 \\
Norway & 54.5 & 95.2 \\
Sweden & 54.7 & 97.0 \\
\hline Netherlands & 46.8 & 98.2 \\
EU-27 & & \\
USA & 55.9 & 67.1 \\
Korea & 28.0 & 83.0 \\
Japan & 31.4 & 55.7 \\
OECD & 37.7 & 79.8 \\
& 28.3 & 90.0 \\
Sourc:Nordic & 30.1 & 77.3 \\
\hline
\end{tabular}

Source: Nordic database, OECD and Eurostat. Åland, Iceland and Japan 2009 data. Netherlands, USA, Korea and OECD: 2008 data. 
Number of pupils in basic school.

\begin{tabular}{lrrrrrrrr}
\hline & \multicolumn{3}{c}{ Primary } & & \multicolumn{3}{c}{ Lower secondary } \\
\cline { 1 - 4 } 1 ooo persons & $\mathbf{2 0 0 0}$ & $\mathbf{2 0 0 5}$ & $\mathbf{2 0 0 9}$ & & $\mathbf{2 0 0 0}$ & $\mathbf{2 0 0 5}$ & $\mathbf{2 0 0 9}$ \\
Denmark & 384 & 414 & 407 & & 207 & 234 & 248 \\
Finland & 388 & 382 & 351 & 198 & 201 & 199 \\
Iceland & 31 & 31 & 30 & 12 & 14 & 14 \\
Norway & 420 & 430 & 427 & 158 & 186 & 189 \\
Sweden & 776 & 719 & 666 & 355 & 419 & 382 \\
Netherlands & 1279 & 1278 & 1290 & & 756 & 789 & 761 \\
EU-27 & 31148 & 28845 & 28082 & 22723 & 23397 & 22066 \\
United States & 24973 & 24455 & 24586 & 12336 & 13166 & 12734 \\
Japan & 7529 & 7232 & 7156 & 4264 & 3689 & 3629
\end{tabular}

Source: Eurostat.

Population projection - children in school age (5-14 years)

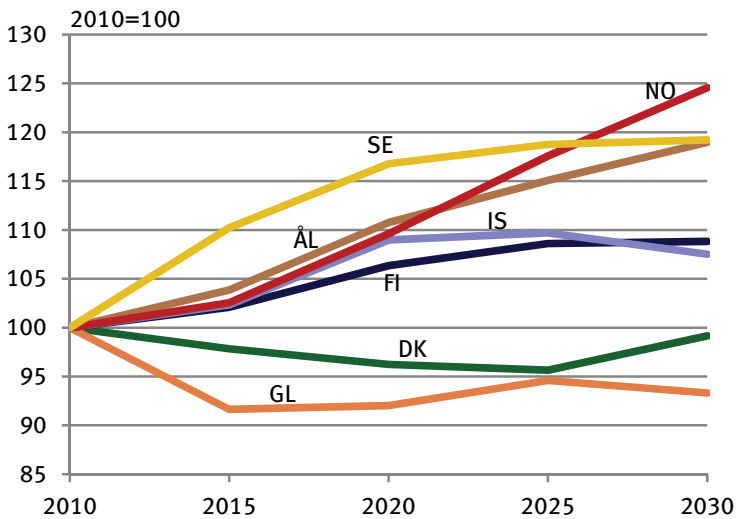

Source: Nordic database.

Nordic Education - Key Data 2012 


\section{Basic school}

Pupils in private primary school

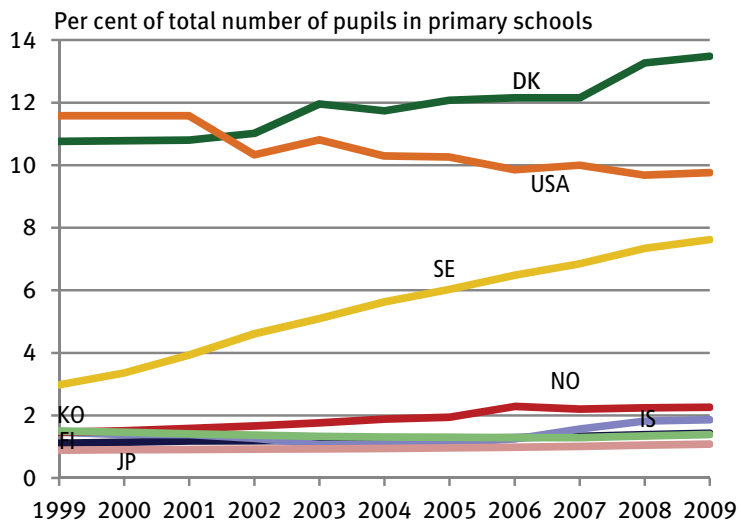

Source: $O E C D$

Pupils in public and private basic school. 2009

$\begin{array}{llllllll}\text { DK } & \text { FI } & \text { IS } & \text { NO } & \text { SE } & \text { KO } & \text { JP } & \text { USA }\end{array}$

Per cent

Primary school

$\begin{array}{lrrrrrrrr}\text { Public } & 86.5 & 98.6 & 98.1 & 97.7 & 92.4 & 98.6 & 98.9 & 90.2 \\ \text { Private } & 13.5 & 1.4 & 1.9 & 2.3 & 7.6 & 1.4 & 1.1 & 9.8 \\ \text { Lower secondary } & & & & & & & & \\ \text { School } & & & & & & & & \\ \text { Public } & 74.2 & 95.6 & 99.2 & 96.9 & 89.7 & 81.6 & 92.8 & 90.9 \\ \text { Private } & 25.8 & 4.4 & 0.8 & 3.1 & 10.3 & 18.4 & 7.2 & 9.1\end{array}$

Source: $O E C D$. 
Average number of pupils per classroom. 2009

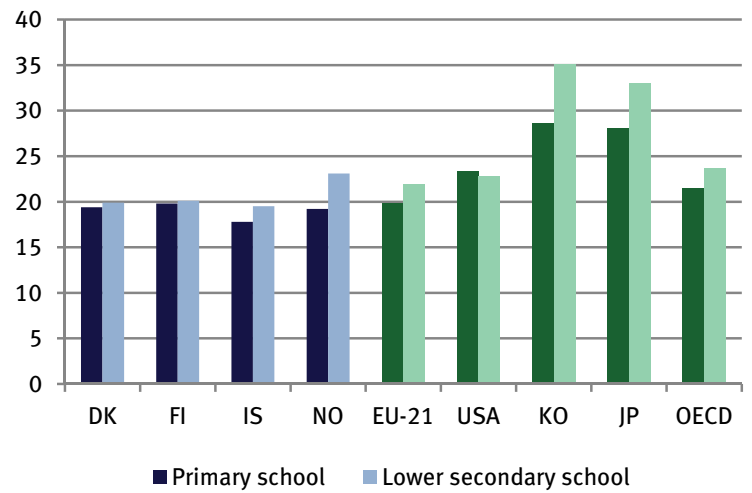

Source: OECD. Norway: 2001 data. Sweden: Data not available. 
Ratio of pupils to teacher staff. 2009

Number of pupils per teacher in full time equivalents

Primary school Lower secondary school

\begin{tabular}{lrr} 
Persons & & \\
Denmark &.. & 9.9 \\
Finland & 13.6 & 10.1 \\
Iceland &.. & 9.9 \\
Norway & 10.7 & 9.9 \\
Sweden & 12.1 & 11.3 \\
\hline Netherlands & 15.8 & \\
EU-21 & 14.5 & 11.5 \\
USA & 14.8 & 14.3 \\
Korea & 22.5 & 19.9 \\
Japan & 18.6 & 14.5 \\
OECD & 16.0 & 13.5 \\
\hline
\end{tabular}

Source: $O E C D$.

Teacher salary after 15 years of experience in lower secondary school. 2009

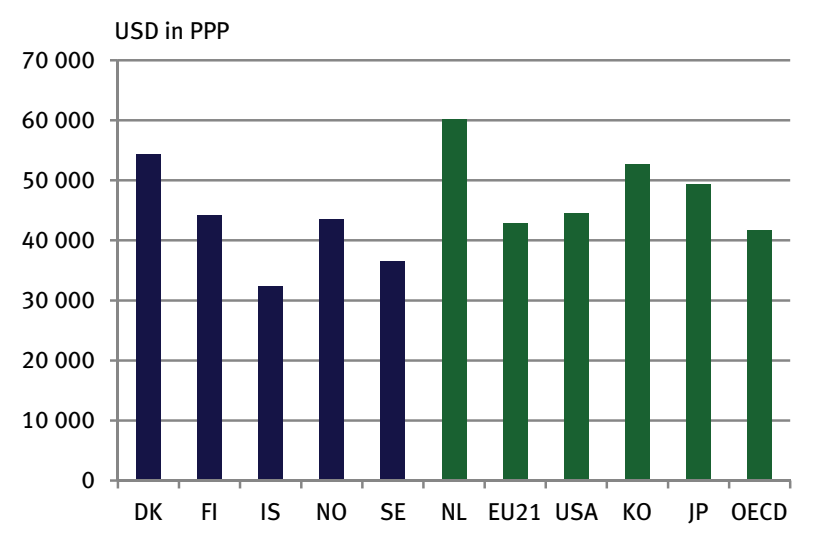

Source: OECD.

Note: The currency is converted from national currencies to USD in Purchasing Power Parities (PPP) to eliminate the impact of differences in price levels among the countries. 
Teachers' working time, primary school. 2009

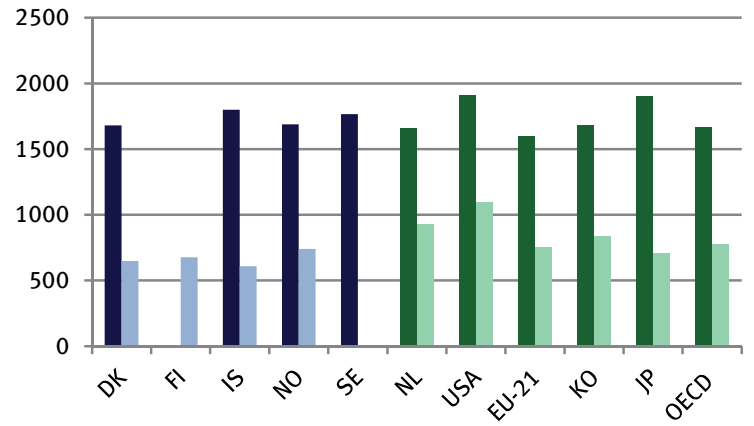

Total working time, hours $\quad$ Net hours spent teaching

Source: $O E C D$

Teachers' working time, primary school. 2009

Average hours Net hours spent

Per cent per year teaching spent teaching

\begin{tabular}{lrrr} 
Denmark & 1680 & 648 & 39 \\
Finland &.. & 677 &.. \\
Iceland & 1800 & 609 & 34 \\
Norway & 1688 & 741 & 44 \\
Sweden & 1767 &.. &.. \\
\hline Netherlands & 1659 & 930 & 56 \\
EU-21 & 1913 & 1097 & 57 \\
USA & 1601 & 763 & 48 \\
Korea & 1680 & 836 & 50 \\
Japan & 1899 & 707 & 37 \\
OECD & 1659 & 779 & 47
\end{tabular}

Source: $O E C D$. 
Basic school/Upper secondary: general and vocational

Instruction in reading, writing and literature, 12-14 year-olds. 2009

Per cent of total compulsory instruction time

25

20

15

10
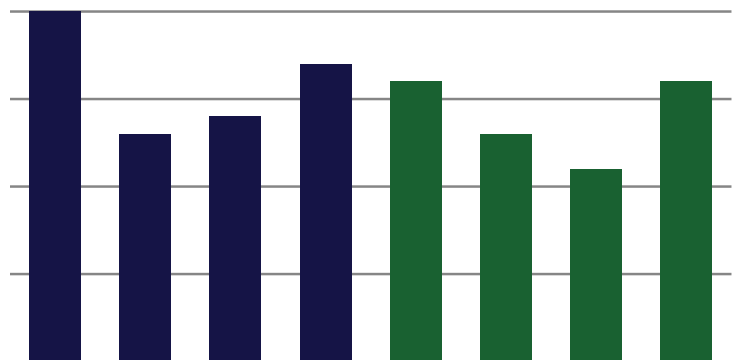

DK

$\mathrm{FI}$

IS

NO

EU21

KO

JP OECD

Source: $O E C D$.

Students in upper secondary education, by orientation. 2009

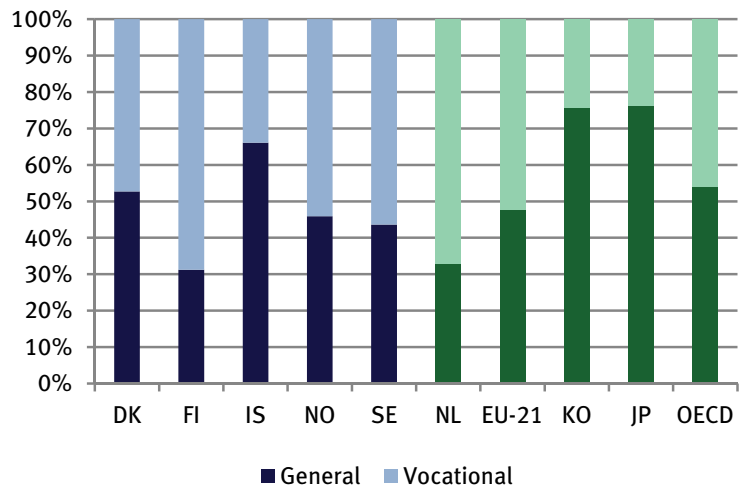

Source: $O E C D$. 


\begin{tabular}{lrrrrr} 
& DK & FI & IS & NO & SE \\
\hline & & & & & \\
Total, 1000s & $\mathbf{2 4 7}$ & $\mathbf{3 9 2}$ & $\mathbf{2 6}$ & $\mathbf{2 6 6}$ & $\mathbf{5 6 4}$ \\
\% Men & 50.9 & 48.2 & 50.1 & 52.8 & 47.4 \\
\% Women & 49.1 & 51.8 & 49.9 & 47.2 & 52.6 \\
\hline & \multicolumn{5}{c}{ Per cent } \\
Total & $\mathbf{1 0 0}$ & $\mathbf{1 0 0}$ & $\mathbf{1 0 0}$ & $\mathbf{1 0 0}$ & $\mathbf{1 0 0}$ \\
General programmes & 34.6 & 28.5 & 64.8 & 39.2 & 26.9 \\
Social science, business and law & 24.0 & 12.9 & 1.5 & 7.5 & 3.7 \\
Engineering, manufacturing and & 23.8 & 22.9 & 13.0 & 21.9 & 17.0 \\
construction & & & & & \\
Health and social services & 7.0 & 11.2 & 3.6 & 9.6 & 5.2 \\
Services and defence & 5.9 & 14.6 & 7.9 & 10.6 & 2.7 \\
Other (incl. not specified) & 4.7 & 9.8 & 9.2 & 11.1 & 44.6 \\
\hline
\end{tabular}

Source: Nordic database. Sweden. 2009 data.

Students at upper secondary level. 2010

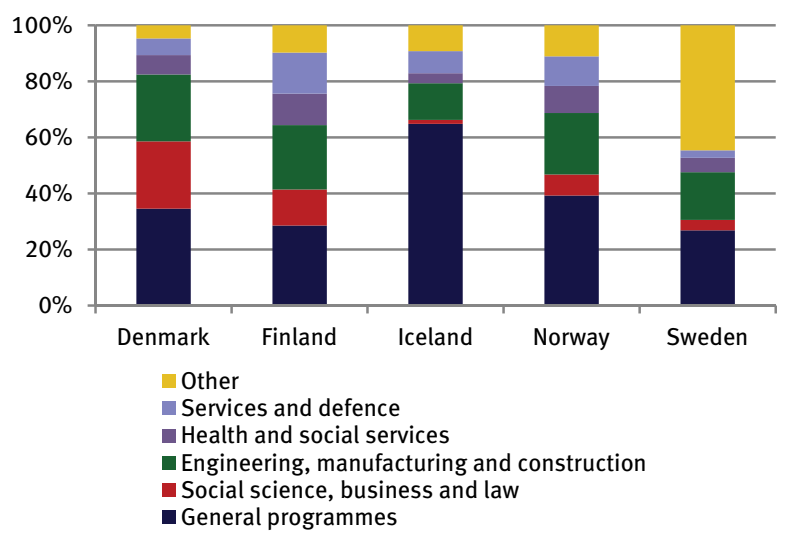

Source: Nordic database. Sweden: 2009 data 


\section{Upper secondary: general and vocational}

\section{Students at upper secondary level}

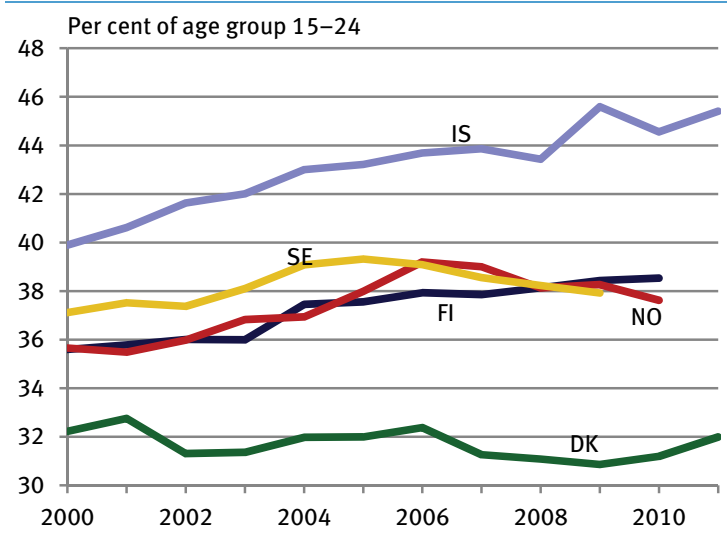

Source: Nordic database.

\section{Graduation rates, upper secondary level. 2009}

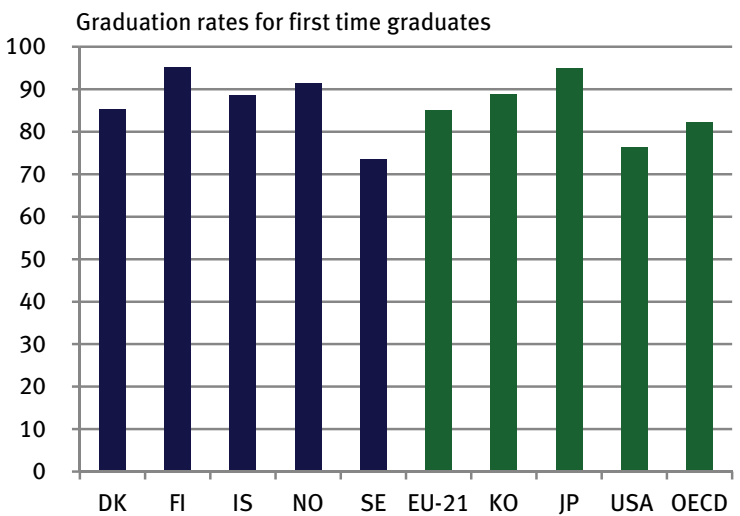

Source: $O E C D$.

Note: Graduation rates: The percentage of the population in the typical age cohort for upper secondary education that successfully completes upper secondary programmes. 
Upper secondary: general and vocational

Student performance by literacy, OECD countries. PISA 2009

\begin{tabular}{|c|c|c|c|c|c|}
\hline \multicolumn{2}{|c|}{ Reading } & \multicolumn{2}{|c|}{ Mathematics } & \multicolumn{2}{|c|}{ Science } \\
\hline Rank & & Rank & & Ran & \\
\hline 1 & Korea, Rep. of & 1 & Korea, Rep. of & 1 & Finland \\
\hline 2 & Finland & 2 & Finland & 2 & Japan \\
\hline 3 & Åland & 3 & Åland & 3 & Åland \\
\hline 6 & Japan & 5 & Japan & 4 & Korea, Rep. of \\
\hline 8 & Netherlands & 7 & Netherlands & 9 & Netherlands \\
\hline 10 & Norway & 13 & Iceland & 18 & United States \\
\hline 14 & Iceland & 14 & Denmark & 19 & OECD \\
\hline 15 & United States & 16 & Norway & 21 & Norway \\
\hline 16 & Sweden & 20 & OECD & 22 & Denmark \\
\hline 20 & Denmark & 22 & Sweden & 24 & Iceland \\
\hline 23 & $\mathrm{OECD}$ & 26 & United States & 25 & Sweden \\
\hline 36 & Faroe Islands & 33 & Faroe Islands & 36 & Faroe Islands \\
\hline
\end{tabular}

Source: $O E C D$

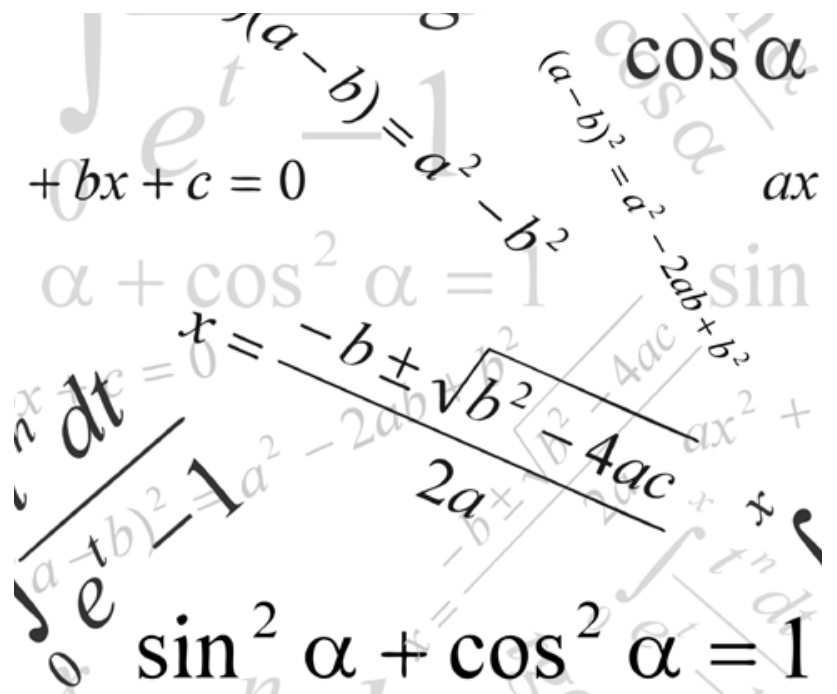




\section{Upper secondary: general and vocational}

\section{Early leavers from education and training}

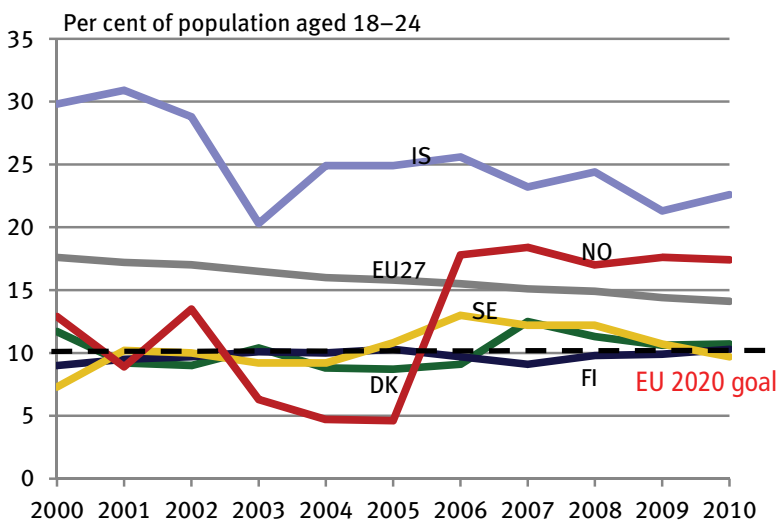

Source: Eurostat

Denmark: Break in time series 2007. Norway: Break in time series 2006. Sweden: Break in time series 2001.

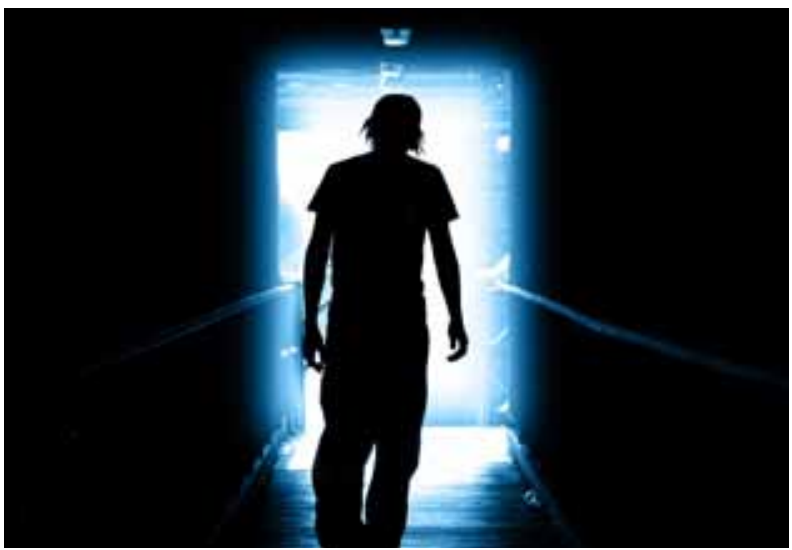




\section{Higher education}

Students at tertiary level. 2010

\begin{tabular}{|c|c|c|c|c|c|}
\hline & DK & $\mathbf{F I}$ & IS & NO & SE \\
\hline Total, $1000 \mathrm{~s}$ & 204 & 308 & 19 & 226 & 469 \\
\hline$\%$ Men & 41.9 & 46.0 & 37.7 & 39.5 & 40.9 \\
\hline \multirow[t]{2}{*}{$\%$ Women } & 58.1 & 54.0 & 62.3 & 60.5 & 59.1 \\
\hline & \multicolumn{5}{|c|}{ Percent } \\
\hline Total & 100 & 100 & 100 & 100 & 100 \\
\hline $\begin{array}{l}\text { Teacher training, education and } \\
\text { science }\end{array}$ & 10.0 & 5.0 & 14.7 & 14.8 & 12.9 \\
\hline Arts, humanities and religion & 16.6 & 14.1 & 14.7 & 10.5 & 13.6 \\
\hline Social science, business and law & 26.3 & 23.0 & 36.0 & 32.3 & 27.5 \\
\hline $\begin{array}{l}\text { Engineering, manufacturing and } \\
\text { construction }\end{array}$ & 10.6 & 23.9 & 9.3 & 8.1 & 16.9 \\
\hline Health and social services & 23.7 & 16.2 & 13.3 & 19.4 & 16.9 \\
\hline Other (incl. not specified) & 12.8 & 17.7 & 12.0 & 14.8 & 12.3 \\
\hline
\end{tabular}

Students at tertiary level. 2010

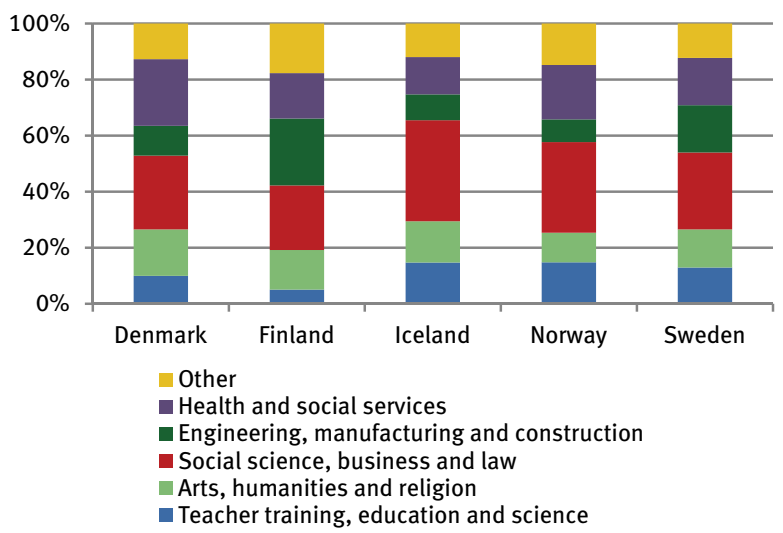

Source: Nordic database. Sweden: 2009 data

Nordic Education - Key Data 2012 


\section{Higher education}

\section{Students at tertiary level}

\section{The educati}

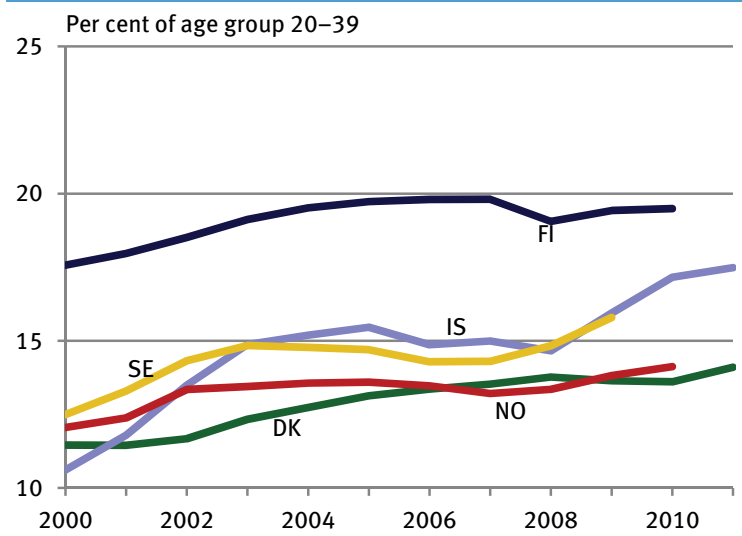

Source: Nordic database.

\section{Women studying at tertiary level}

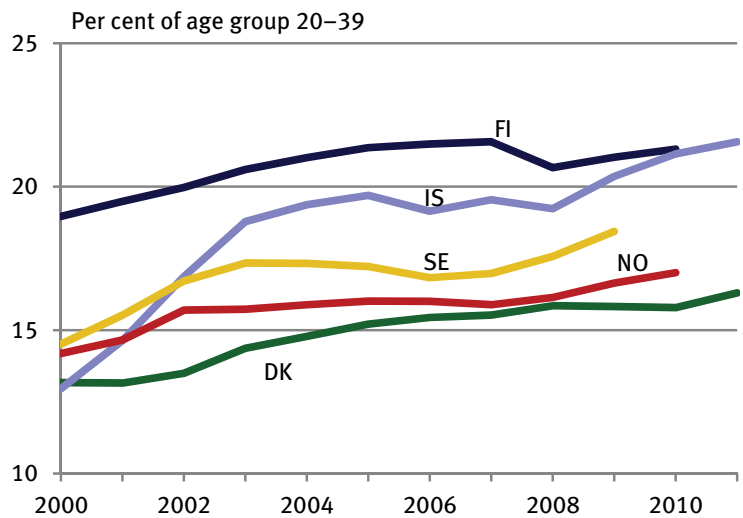

Source: Nordic database 


\section{Men studying at tertiary level}

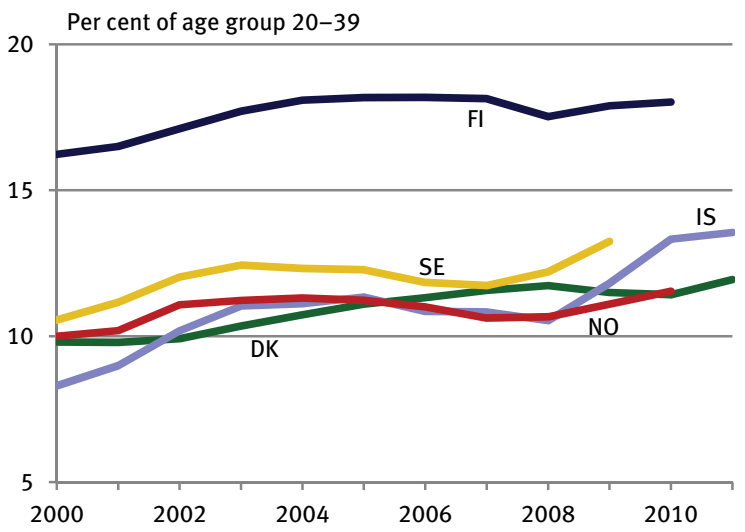

Source: Nordic database.

\section{Graduates from tertiary level}

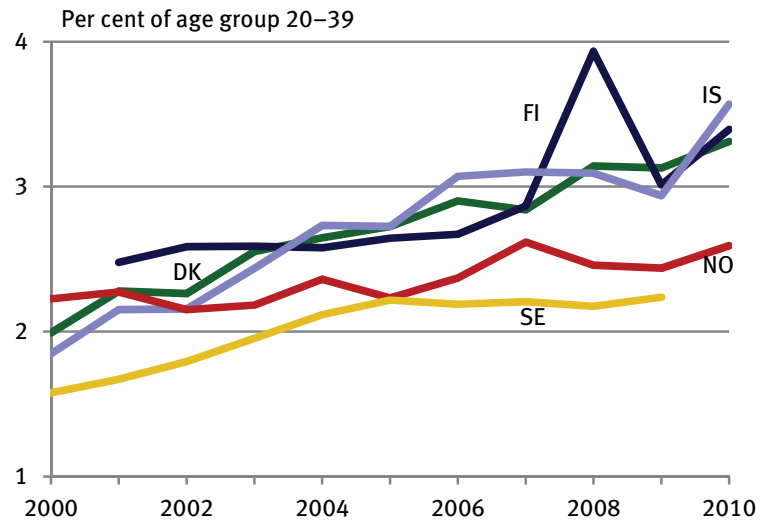

Source: Nordic database. 


\section{Higher education/Students abroad}

\section{Graduation rates at tertiary level}

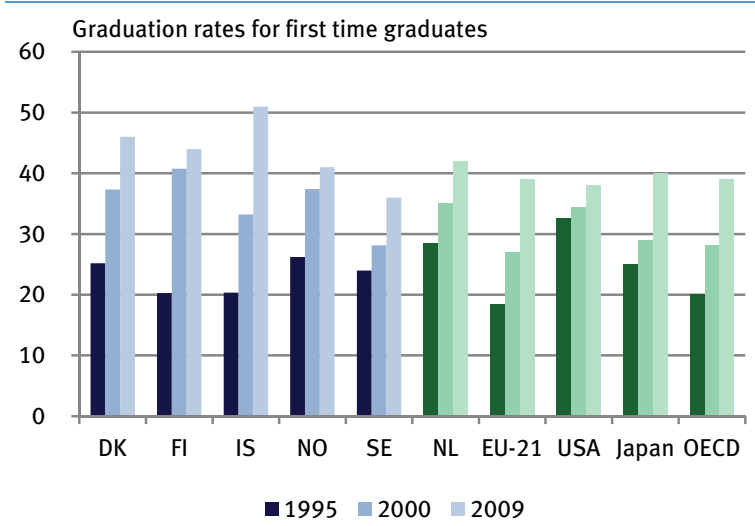

Source: $O E C D$.

Note: Graduation rates: The percentage of the population in the typical age cohort for tertiary education that successfully completes tertiary programmes.

\section{Nordic students in the other Nordic countries by type of study. 2010/11}

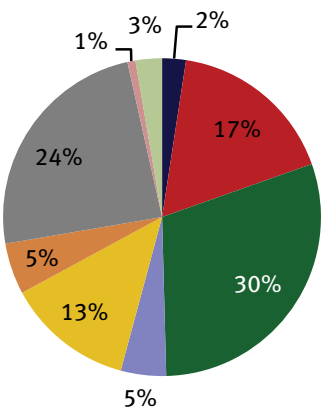

- Teacher training, education science

Arts, humanities and religion

- Social science, business and law

- Natural science, mathematics and computing

Engineering, manufacturing and construction

- Agriculture forestry and fishery

- Health and social services

Services and defence

Field not specified

Source: Kela - The Social Insurance Institution of Finland.

Note: All data related to students abroad is limited to students registered as receiving financial support for studies abroad. Covers full-time students taking exams abroad. 
Nordic students in the other Nordic countries. 2010/11

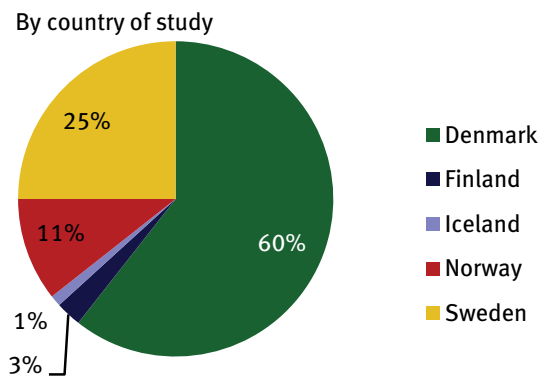

Source: Nordic database.

Nordic students in the other Nordic countries. 2010/11

\begin{tabular}{lrrrrrr}
\hline Students from & DK & FI & IS & NO & SE & Nordic \\
\hline Total & $\mathbf{6 1 4}$ & $\mathbf{1 1 7 8}$ & $\mathbf{1 3 0 7}$ & $\mathbf{4 0 8 7}$ & $\mathbf{3 1 2 1}$ & $\mathbf{1 0 3 0 7}$ \\
Denmark &. & 133 & 1058 & 2938 & 2117 & 6246 \\
Finland & 16 &. & 3 & 37 & 215 & 271 \\
Iceland & 46 & 13 &. & 34 & 26 & 119 \\
Norway & 189 & 76 & 66 &. & 763 & 1094 \\
Sweden & 363 & 956 & 180 & 1078 &. & 2577 \\
\hline
\end{tabular}

Source: Nordic database.

Note: Data limited to students registered as receiving financial support for studies abroad. Covers full-time students taking exams abroad. 
Nordic students abroad. 2010/11

\begin{tabular}{lrrrrrr}
\hline Students from & DK & FI & IS & NO & SE & Nordic \\
\hline Total & $\mathbf{3 3 8 5}$ & $\mathbf{5 4 5 7}$ & $\mathbf{2 4 0 6}$ & $\mathbf{1 5} \mathbf{1 6 9}$ & $\mathbf{2 1 7 0 5}$ & $\mathbf{4 8 1 2 2}$ \\
\hline Country of study: & & & & & & \\
Nordic total & 614 & 1178 & 1307 & 4087 & 3121 & 10307 \\
Baltic countries & 2 & 558 & 3 & 82 & 165 & 810 \\
France & 69 & 101 & 23 & 322 & 832 & 1347 \\
Germany & 198 & 263 & 61 & 207 & 656 & 1385 \\
Great Britain & 1478 & 1777 & 247 & 3552 & 4484 & 11538 \\
Netherlands & 126 & 215 & 100 & 361 & 241 & 1043 \\
Spain & 71 & 113 & 49 & 189 & 1174 & 1596 \\
Hungary & 9 & 21 & 77 & 751 & 428 & 1286 \\
Poland & 21 & 16 & 4 & 1383 & 1100 & 2524 \\
Other Europe & 125 & 395 & 105 & 989 & 2038 & 3652 \\
USA, Canada and & & & & & & \\
other America & 343 & 416 & 381 & 1472 & 4583 & 7195 \\
Asia & 15 & 94 & 17 & 163 & 1384 & 1673 \\
Australia, New & & & & & & \\
Zealand and & & & & & & \\
other Oceania & 131 & 159 & 29 & 1511 & 1354 & 3184 \\
Other & 183 & 151 & 3 & 100 & 145 & 582 \\
\hline
\end{tabular}

Source: Nordic database.

\section{Nordic students abroad. 2010/11}

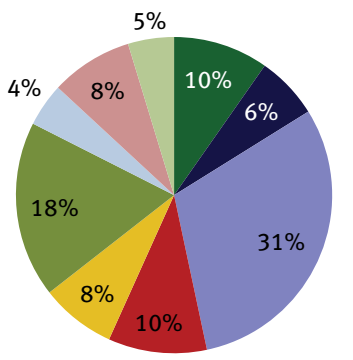

- France, Italy and Spain

- Germany and the Netherlands

- Great Britain

- Hungary and Poland

Other Europe

- USA and Canada

Asia

Australia and New Zealand Other

Source: Nordic database. 
Attained level of education. Population aged 15-74. 2011

\begin{tabular}{|c|c|c|c|c|c|}
\hline & $\begin{array}{r}\text { Total } \\
(\text { ISCED } \\
1-6)\end{array}$ & $\begin{array}{r}\text { Primary } \\
\text { level } \\
(I S C E D \\
1-2)\end{array}$ & $\begin{array}{r}\text { Secondary } \\
\text { level } \\
(I S C E D \\
3-4)\end{array}$ & $\begin{array}{r}\text { Tertiary } \\
\text { level } \\
(I S C E D \\
5-6)\end{array}$ & $\begin{array}{r}\text { Level not } \\
\text { specified } \\
\text { or } \\
\text { unknown }\end{array}$ \\
\hline \multicolumn{6}{|l|}{ Per cent } \\
\hline \multicolumn{6}{|l|}{ Total } \\
\hline Denmark & 100 & 30.5 & 40.4 & 25.3 & 3.8 \\
\hline Finland & 100 & 29.0 & 41.7 & 29.4 & 0.0 \\
\hline Åland & 100 & 37.4 & 39.3 & 23.3 & 0.0 \\
\hline Iceland & 100 & 38.3 & 35.5 & 26.0 & 0.3 \\
\hline Norway & 100 & 26.0 & 40.1 & 27.6 & 6.3 \\
\hline Sweden & 100 & 21.3 & 50.3 & 26.3 & 2.1 \\
\hline \multicolumn{6}{|l|}{ Men } \\
\hline Denmark & 100 & 30.6 & 43.0 & 22.5 & 3.9 \\
\hline Finland & 100 & 31.0 & 43.8 & 25.3 & 0.0 \\
\hline Åland & 100 & 39.5 & 38.4 & 22.2 & 0.0 \\
\hline Iceland & 100 & 35.6 & 42.1 & 22.0 & 0.4 \\
\hline Norway & 100 & 26.4 & 42.3 & 24.6 & 6.8 \\
\hline Sweden & 100 & 23.0 & 52.6 & 22.1 & 2.3 \\
\hline \multicolumn{6}{|l|}{ Women } \\
\hline Denmark & 100 & 30.4 & 37.8 & 28.2 & 3.6 \\
\hline Finland & 100 & 27.0 & 39.5 & 33.6 & 0.0 \\
\hline Åland & 100 & 35.2 & 40.3 & 24.5 & 0.0 \\
\hline Iceland & 100 & 41.0 & 28.7 & 30.1 & 0.3 \\
\hline Norway & 100 & 25.6 & 37.8 & 30.8 & 5.9 \\
\hline Sweden & 100 & 19.6 & 47.9 & 30.6 & 1.9 \\
\hline
\end{tabular}

Source: Nordic database. Finland, Åland and Norway: 2010 data.

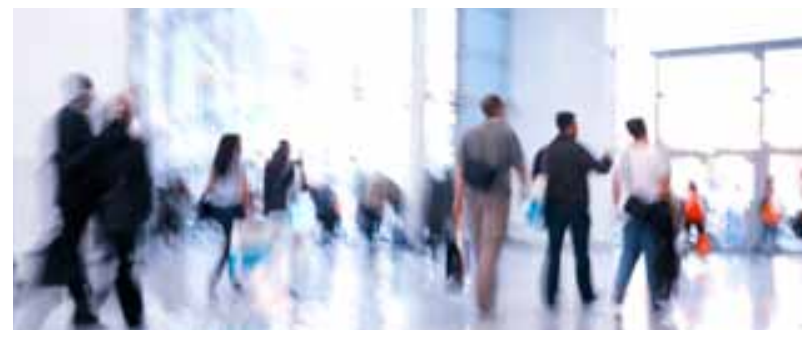




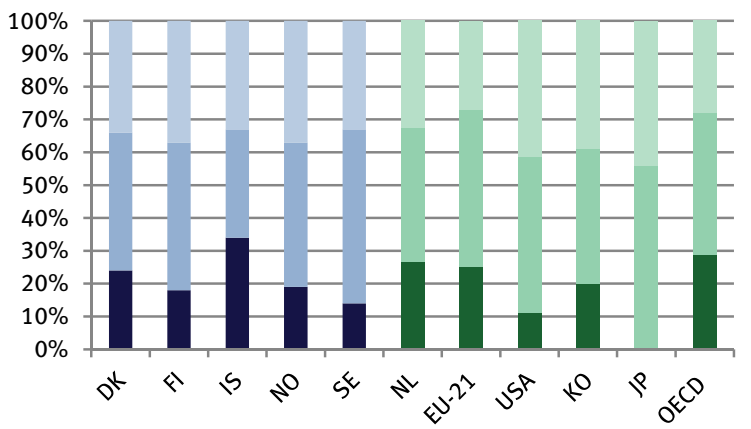

घ Primary level $\square$ Upper secondary level $\square$ Tertiary level

Source: $O E C D$

\section{Tertiary level attained. Population aged 30-34}

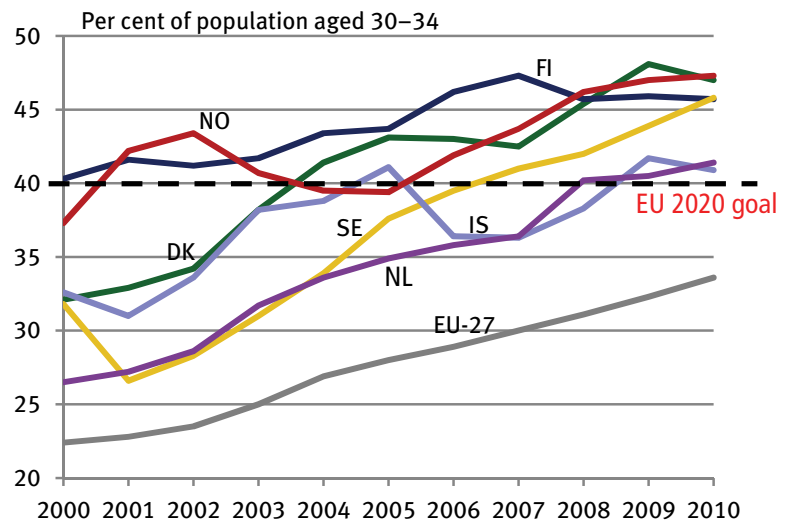

Source: Eurostat 
Attained education. Population aged 25-64 years

\begin{tabular}{|c|c|c|c|c|c|c|c|c|c|}
\hline & \multicolumn{3}{|c|}{$\begin{array}{c}\text { Primary } \\
\text { Level }\end{array}$} & \multicolumn{3}{|c|}{$\begin{array}{c}\text { Upper } \\
\text { secondary level }\end{array}$} & \multicolumn{3}{|c|}{$\begin{array}{c}\text { Tertiary } \\
\text { level }\end{array}$} \\
\hline & 2000 & 2005 & 2009 & 2000 & 2005 & 2009 & 2000 & 2005 & 2009 \\
\hline \multicolumn{10}{|l|}{ Per cent } \\
\hline Denmark & 21 & 19 & 24 & 52 & 47 & 42 & 26 & 34 & 34 \\
\hline Finland & 27 & 21 & 18 & 41 & 44 & 45 & 32 & 35 & 37 \\
\hline Iceland & 45 & 37 & 34 & 32 & 32 & 33 & 23 & 31 & 33 \\
\hline Norway & 15 & 23 & 19 & 15 & 23 & 19 & 28 & 33 & 37 \\
\hline Sweden & 22 & 16 & 14 & 47 & 54 & 53 & 30 & 30 & 33 \\
\hline Netherlands & 35 & 28 & 27 & 41 & 42 & 41 & 23 & 30 & 33 \\
\hline EU-21 & 36 & 29 & 25 & 45 & 48 & 48 & 19 & 24 & 27 \\
\hline USA & 13 & 12 & 11 & 51 & 48 & 47 & 36 & 39 & 41 \\
\hline Korea & 32 & 24 & 20 & 44 & 44 & 41 & 24 & 32 & 39 \\
\hline Japan & 17 & .. & .. & 49 & 60 & 56 & 34 & 40 & 44 \\
\hline OECD & 36 & 30 & 27 & 43 & 44 & 44 & 22 & 27 & 30 \\
\hline
\end{tabular}

Source: $O E C D$.

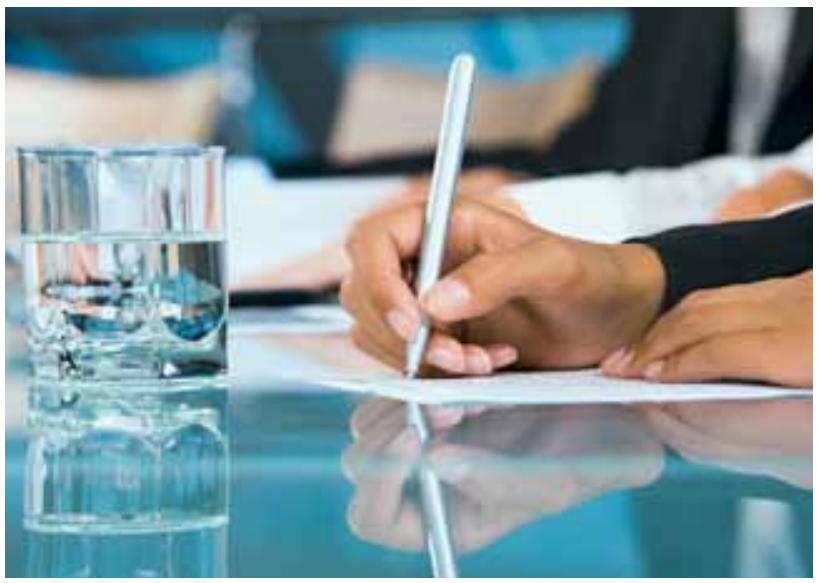


Participation in formal or non-formal education after leaving initial education. 2007

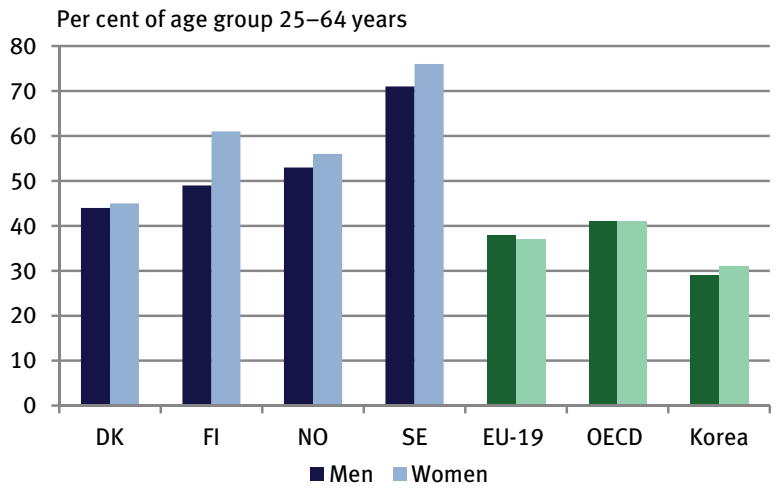

Source: Eurostat.

Participation in formal or non-formal education after leaving initial education, by attained education. 2007

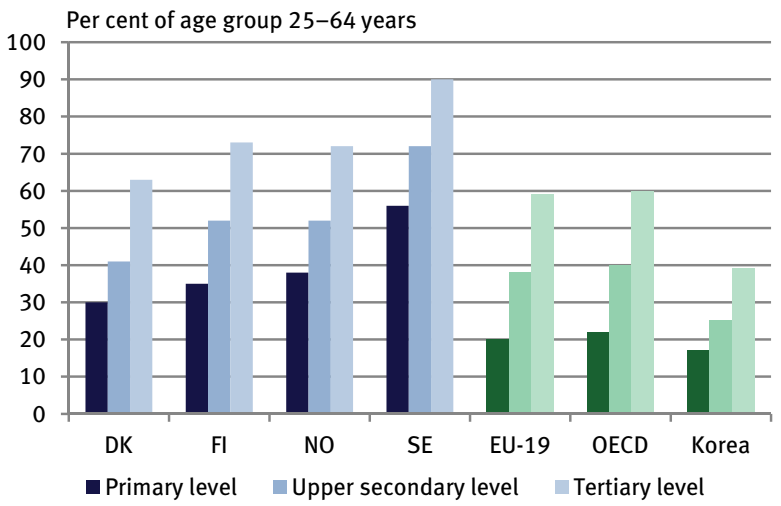

Source: Eurostat. 


\section{Public expenditure on education}

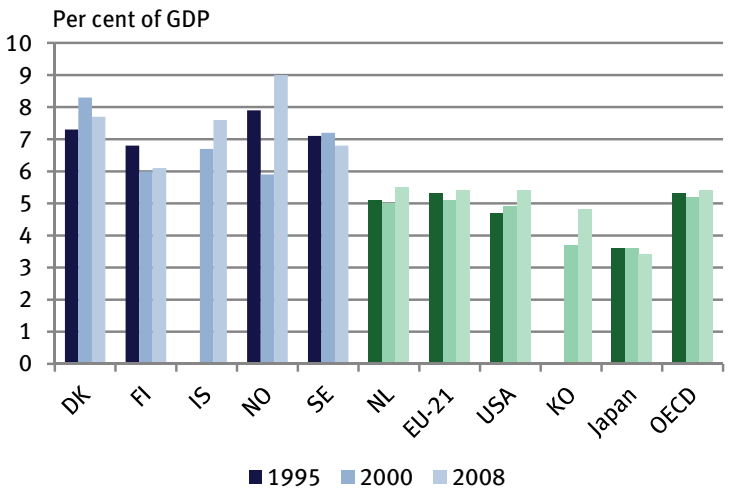

Source: $O E C D$

\section{Expenditure on educational institutions. 2008}

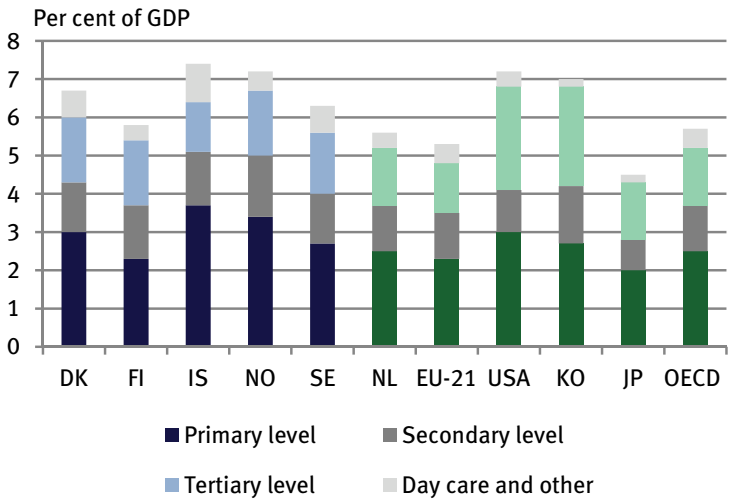

Source: $O E C D$

Note: Norway: Government expenditure only 


\section{Primary education}

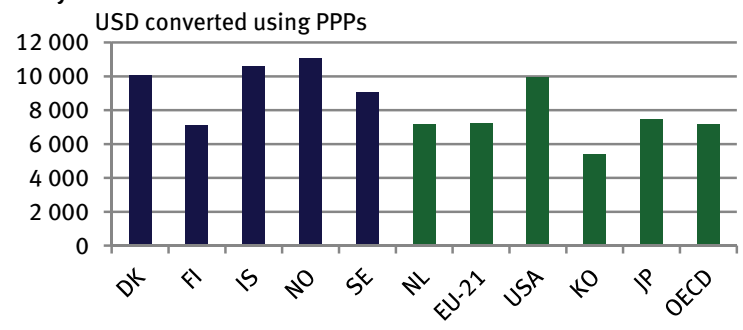

\section{Secondary education}

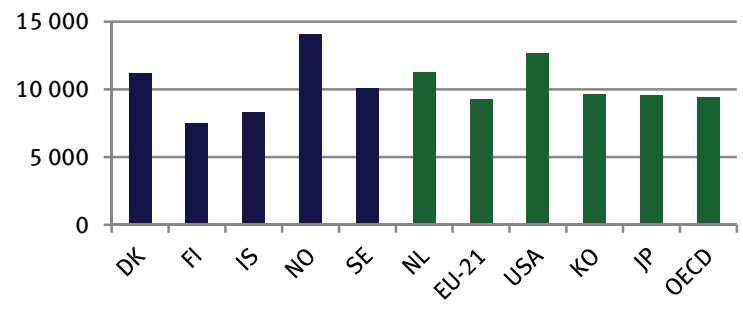

Tertiary education

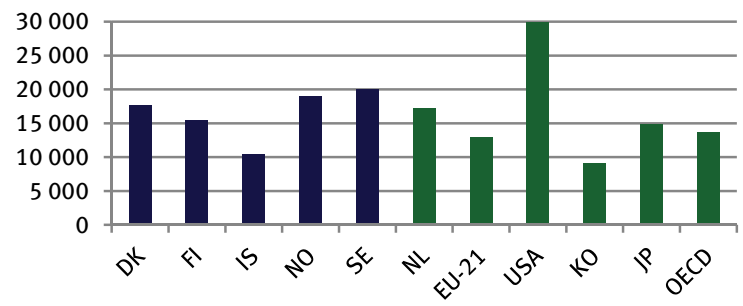

Source: $O E C D$

Note: The currency is converted from national currencies to USD in Purchasing Power Parities (PPP) to eliminate the impact of differences in price levels amona the countries. 
Expenditure in the education system

Expenditure on educational institutions by financing source. 2008

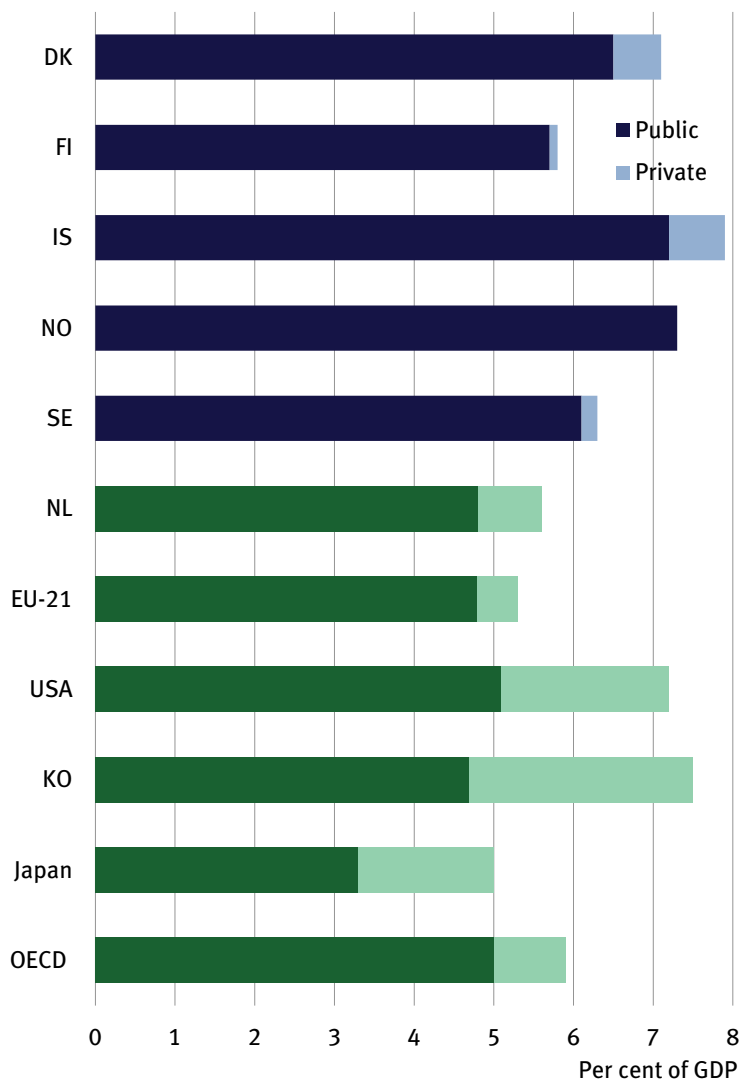

Source: $O E C D$. 


\section{The nordic education systems}

\section{The Danish education system}

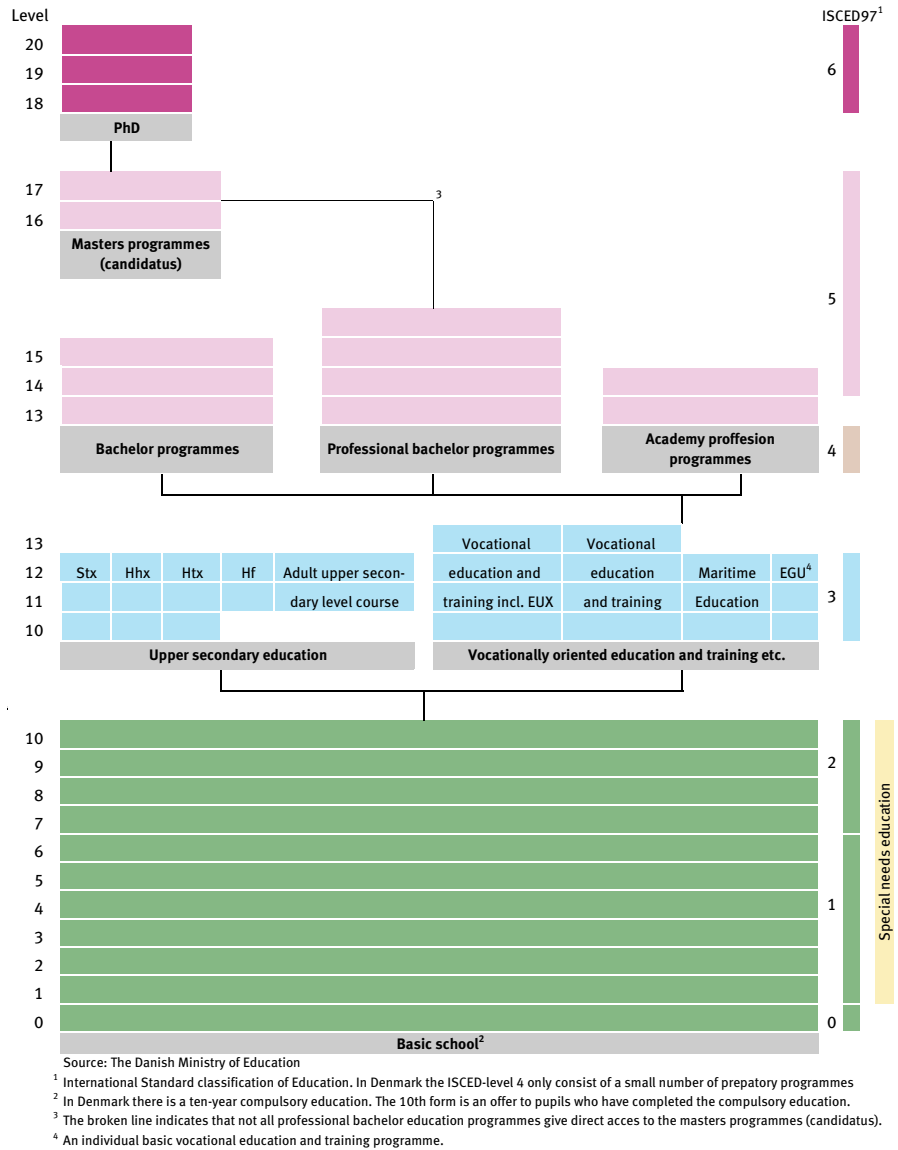




\section{The Finnish education system}

ISCED-

classification

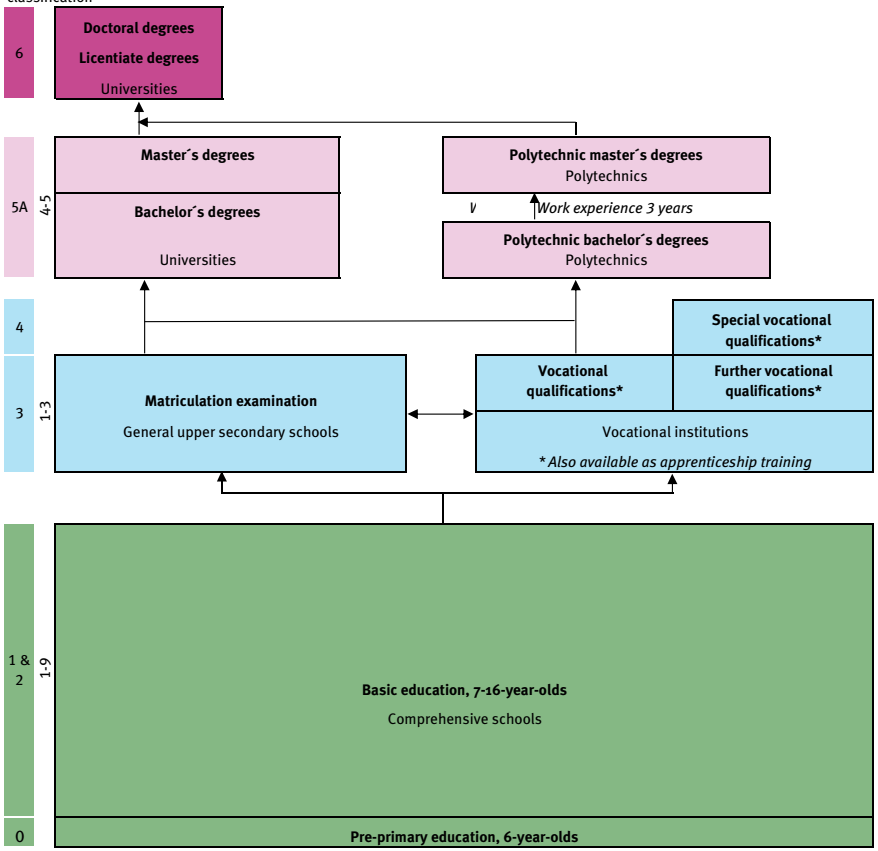

S Source: Ministry of Education and Culture, Finland

ISCED-classification 1997

0 Pre primary education

1-2 Primary education or lower secondary education

4 Post-scondary non-tertiary education

3 Upper sccondary education

5 First stage of tertiary education

6 Second stage of tertiary education 


\section{The nordic education systems}

\section{The Icelandic education system}

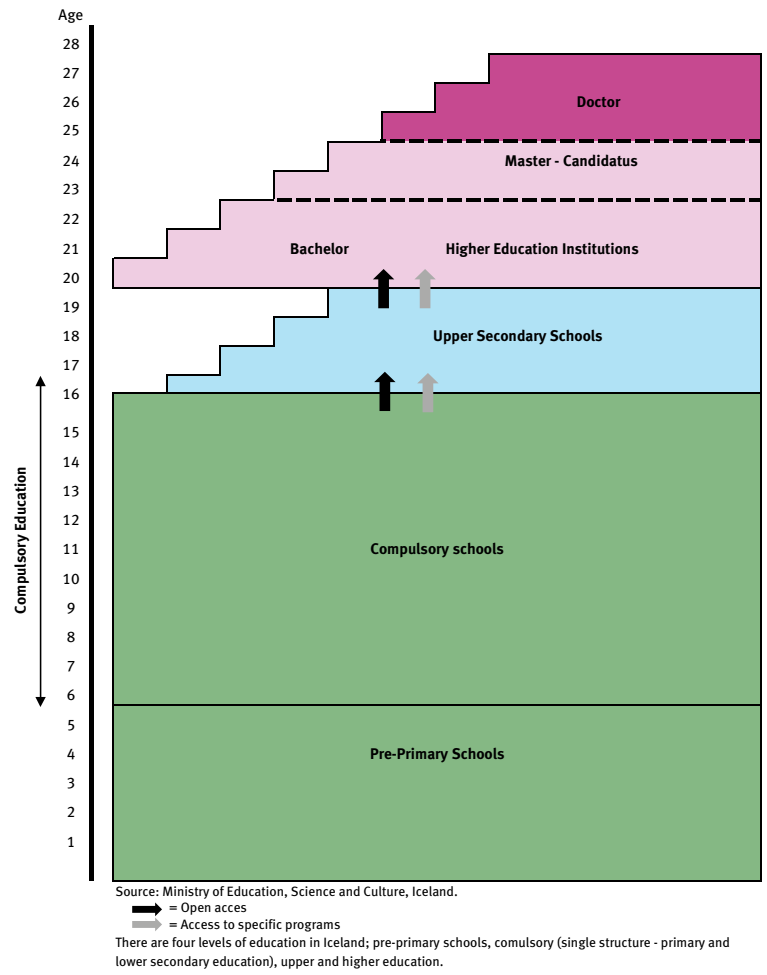




\section{The Norwegian education system}

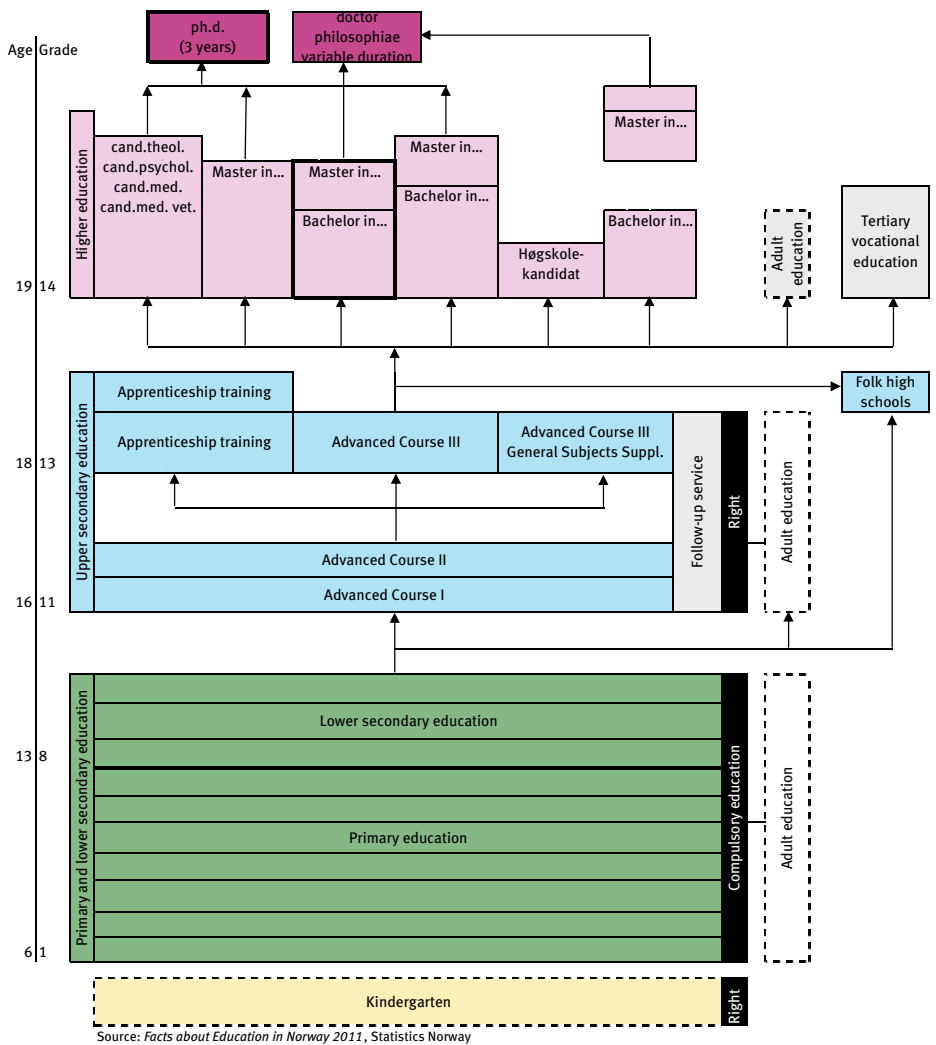




\section{The nordic education systems}

\section{The Swedish education system}

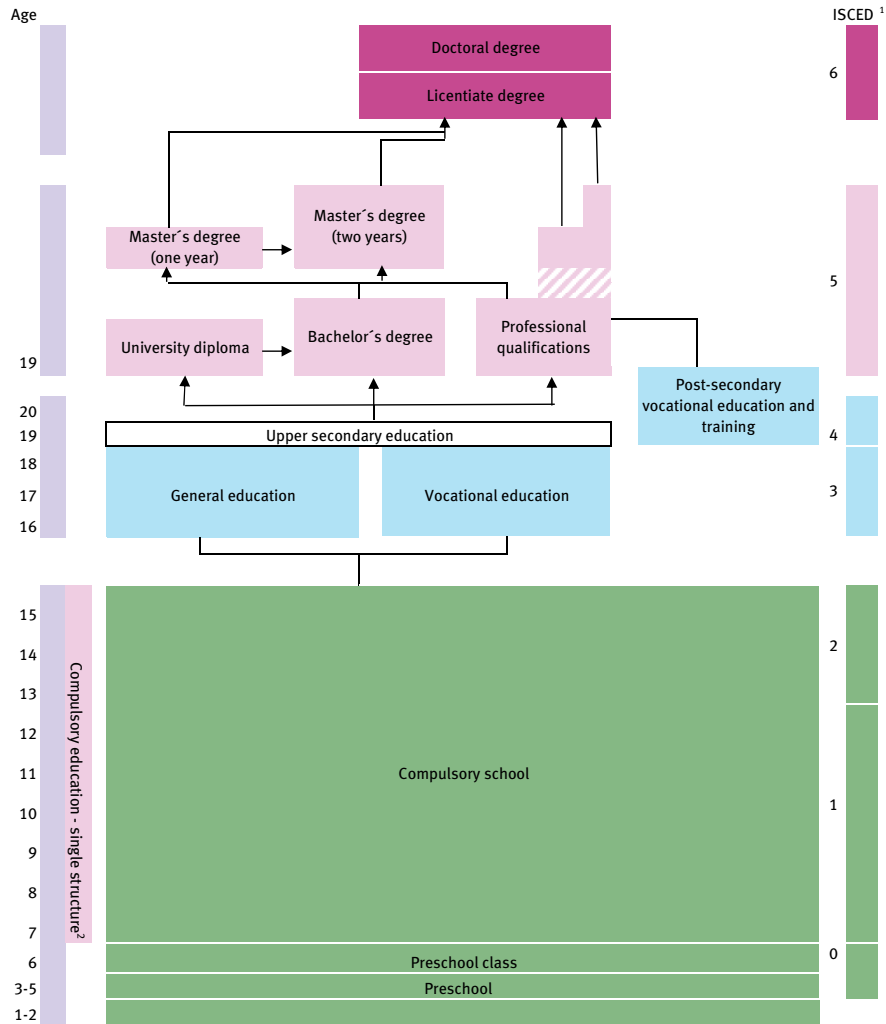

Source: Based on European Comission, Structures of education and training systems in Europe. Sweden. 2009/10 Edition and Swedish National Agency for Higher Education.

${ }^{1}$ International Standard classification of Education

${ }^{2}$ No institutional distinction between ISCED 1 and 2 . 



\section{nordan}

Nordic Council of Ministers

Ved Stranden 18

DK-1061 Copenhagen K

www.norden.org

ISBN 978-92-893-2367-3

DOI http://dx.doi.org/10.6027/ANP2012-733 ANP 2012:733 